\title{
CircRNA_0092516 regulates chondrocyte proliferation and apoptosis in osteoarthritis through the miR-337-3p/PTEN axis
}

\section{Zhihui Huang}

the third affiliated hospital of suchow university,changzhou

\section{Wenming $\mathrm{Ma}$}

the third affiliated hospital of suchow university

Jinhuai Xiao

the third affiliated hospital of suchow university,changzhou

Xiaoyu Dai

the third affiliated hospital of suchow university,changzhou

Weiqi Ling ( $\nabla$ Weiqilingwql@126.com )

the third affiliated hospital of suchow university,changzhou

\section{Research}

Keywords: circRNA_0092516, miR-337-3p, PTEN, osteoarthritis, chondrocyte proliferation, apoptosis

Posted Date: July 29th, 2020

DOI: https://doi.org/10.21203/rs.3.rs-50001/v1

License: (c) (1) This work is licensed under a Creative Commons Attribution 4.0 International License.

Read Full License

Version of Record: A version of this preprint was published at The Journal of Biochemistry on November 2nd, 2020. See the published version at https://doi.org/10.1093/jb/mvaa119. 


\section{Abstract \\ Background}

The dysregulation of circular RNAs (circRNAs) has been identified in various human diseases. Here, we mainly investigated the potential mechanism of circRNA_0092516 in osteoarthritis (OA).

\section{Methods}

The expression of circRNA_0092516 was detected by quantitative real-time PCR. MTT, flow cytometry, and Western blot were used to confirm the functions of circRNA_0092516 in vitro. Besides, RNA pull-down and dual-luciferase reporter gene experiments were used to study the potential mechanism.

\section{Results}

circRNA_0092516 was up-regulated in the tissues of OA patients and chondrocytes stimulated by IL-1 $\beta$. The potential mechanism analysis revealed that circRNA_0092516 bound to miR-337-3p, and the interference with circRNA_0092516 promoted chondrocyte proliferation and repressed cell apoptosis through the miR-337-3p/PTEN axis, thereby improving OA.

\section{Conclusions}

Overall, our data showed that the interference with circRNA_0092516 promoted chondrocyte proliferation and repressed cell apoptosis through the miR-337-3p/PTEN axis, eventually slowed down the progress of OA.

\section{Introduction}

Osteoarthritis (OA) is a type of age-related joint disease that has been widely regarded as an irreversible disease and one of the main causes of disability in the elderly[1]. Although the clinical treatment of OA has made good progress, there are still deficiencies such as poor prognosis and expensive treatment costs[2, 3]. During the occurrence of $\mathrm{OA}$, various pathological factors directly or indirectly act on chondrocytes, and emerging evidence has confirmed that the inhibition of chondrocyte proliferation, differentiation, and inflammatory response are conducive to hindering the occurrence and development of $\mathrm{OA}[4,5]$. Therefore, we urgently need to explore the underlying mechanism of chondrocyte proliferation and apoptosis in $O A$, which is critical for the relief of OA.

Recently, circular RNAs (circRNAs) have gradually become a popular molecule in non-coding RNA research and is characterized by a stable structure and high tissue-specific expression[6, 7]. Increasing evidence indicates that the dysregulation of circRNAs is closely related to the progress of various human 
diseases. For instance, Zhao et al. found that circ_0136474 is significantly up-regulated in OA cartilage tissues, and this highly expressed circ_0136474 inhibits chondrocyte proliferation and promotes cell apoptosis[8]. Shen et al. indicated that the overexpression of circSERPINE2 impedes the progression of OA by inhibiting human chondrocyte apoptosis[9]. Here, what attracts our attention is that circRNA_0092516, also known as circRNA-NT5C2, has been reported to be up-regulated in OA[10], indicating that circRNA_0092516 might be related to the occurrence and development of OA. Besides, increasing evidence shows that circRNAs are rich in the binding sites of miRNAs, and affect the expression of downstream target mRNA by competing for endogenous RNA (ceRNA) to interact with miRNA[11, 12]. However, it is currently unclear whether circRNA_0092516 can act as a ceRNA in the progress of $O A$.

MicroRNAs (miRNAs) are small non-coding RNAs that cannot encode proteins, and negatively regulate target mRNAs expression by interacting with the 3 'untranslated region (UTR) of the target mRNAs[13]. Various previous studies have shown that the abnormal expressions of miRNAs are closely related to the development of $O A[14,15]$. As reported, after the occurrence of OA, miR-181a-5p is abnormally downregulated and the in-depth mechanism research finds that miR-181a-5p plays an important role in the process of OA by negatively regulating SBP2[16]. Besides, Chen et al. found that miR-29b-3p promotes chondrocyte apoptosis and promotes the occurrence and development of OA through targeting PGRN[17]. Importantly, our previous study shows that miR-337-3p is significantly down-regulated in OA cartilage tissues[18]. However, the mechanism by which miR-337-3p plays roles in OA has not been fully elucidated.

In the current study, we mainly explored the role and potential mechanism of circRNA_0092516 in OA.

\section{Materials And Methods}

\subsection{Patients and tissue samples}

10 cases of osteoarthritis cartilage tissue were collected from patients with knee OA of total knee replacement surgery, and 10 cases of normal articular cartilage tissues were obtained from nonosteoarthritis patients who had undergone surgical repair of knee fractures and had no history of arthritis. All cartilage donors included in this study provided informed consent. This study was approved by the Human Ethics Committee of the Third Affiliated Hospital of Suchow University.

\subsection{RNA extraction and quantitative real-time PCR (qRT- PCR)}

qRT-PCR experiments were performed according to the previously described method with minor changes[19]. TRIzol reagent was used to isolate the total RNA from cartilage tissues and primary chondrocytes, and then RevertAid First Strand cDNA Synthesis Kit (Thermo Scientific) was used to reverse transcribe RNA into cDNA. Next, Forget-Me-Not ${ }^{\text {TM }}$ EvaGreen ${ }^{\circledR}$ qPCR Master Mix (Biotium) and CFX96 ${ }^{\text {TM }}$ Real-Time PCR Detection Systems (BIO-RAD) were used to perform the real-time PCR. GAPDH 
was used as an internal control for circRNA_0092516, and U6 was used as an internal control for miR337-3p. Finally, the relative expressions of circRNA_0092516 and miR-337-3p were quantified using the $2^{-\triangle \Delta C t}$ method.

\subsection{Isolation of primary chondrocytes}

Cartilage slices were obtained under sterile conditions and cut into thin slices, then placed them in phosphate-buffered saline (PBS) supplemented with $1 \%$ trypsin at $37^{\circ} \mathrm{C}$ for $15 \mathrm{~min}$, and then centrifuged to discard the supernatant. We washed the collected cartilage 3 times to remove trypsin and continued to incubate for $12-16 \mathrm{~h}$ at $37^{\circ} \mathrm{C}$ in PBS supplemented with $2 \mathrm{mg} / \mathrm{L}$ collagenase IV. Next, the above chondrocytes were washed with Dulbecco's Modified Eagle's medium (DMEM) supplemented with penicillin $(100 \mathrm{U} / \mathrm{mL})$-streptomycin $(100 \mu \mathrm{g} / \mathrm{mL})$ and centrifuged at $200 \mathrm{~g}$ for $10 \mathrm{~min}$ to harvest the final primary chondrocytes.

\subsection{Culture and different treatment of primary chondrocytes}

The primary chondrocytes harvested above were placed in DMEM medium supplemented with $100 \mathrm{U} / \mathrm{mL}$ penicillin, $100 \mu \mathrm{g} / \mathrm{mL}$ streptomycin, $1 \%$ glutamine, and $10 \%$ fetal bovine serum (FBS), and cultured at $37^{\circ} \mathrm{C}, 5 \% \mathrm{CO}_{2}$. Besides, we added $10 \mathrm{ng} / \mathrm{mL} \mathrm{IL}-1 \beta$ to the DMEM medium to stimulate primary chondrocytes for $24 \mathrm{~h}$.

\subsection{Western blot analysis}

RIPA lysis buffer was used to extract total protein from cartilage tissues and primary chondrocytes, and the BCA Protein Assay Kit (Sigma) was used to quantify the concentration of the above-extracted protein. Then, the same amount of protein was separated on $10 \%$ SDS-polyacrylamide gel electrophoresis (SDSPAGE) and then transferred to a PVDF membrane (Millipore). After blocking with $5 \%$ skim milk, the membrane was incubated with the primary antibody overnight at $4{ }^{\circ} \mathrm{C}$. The next day, the membrane and the secondary antibody were incubated at room temperature for $1 \mathrm{~h}$. Finally, according to the standard procedures of the manufacturer, the ECL chemiluminescence kit (Santa Cruz Biotechnology) was used to detect the protein, and the image was processed using ImageJ software.

\subsection{Cell transfection}

Briefly, primary chondrocytes were cultured in 96-well plates supplemented with DMEM. According to the reagent manufacturer's instructions, the synthetic si-circRNA_0092516, si-circRNA_0092516 + miR-337-3p inhibitor were transfected into primary chondrocytes using Lipofectamine 2000 (Invitrogen). $48 \mathrm{~h}$ after transfection, the cells were harvested for subsequent research.

\subsection{Detection of cell proliferation}

According to the manufacturer's standard protocol, the proliferation of primary chondrocytes was analyzed using the MTT Assay kit (Abcam). Briefly, primary chondrocytes with different treatments were seeded in 96-well plates and cultured for $48 \mathrm{~h}$. Next, $20 \mu \mathrm{L}$ of MTT solution was added to each well and 
incubated at $37^{\circ} \mathrm{C}$ for another $2 \mathrm{~h}$. Then, the absorbance was measured at a wavelength of $570 \mathrm{~nm}$ to determine the proliferation of primary chondrocytes.

\subsection{Flow cytometry assay}

Based on the previously described methods[20], we performed the flow cytometry assay to detect the apoptosis of chondrocytes. After washing with cold phosphate buffer, the primary chondrocytes after different treatments were collected. Then, the cells were resuspended in the membrane conjugate buffer and incubated with $5 \mu \mathrm{L}$ LITC Annexin V and $5 \mu \mathrm{L}$ PI working solution for $15 \mathrm{~min}$ at room temperature. Finally, the flow cytometry FACSCalibur instrument (BD Biosciences) was used to analyze the apoptosis ratio of primary chondrocytes.

\subsection{Dual-luciferase reporter gene assay}

293T cells $\left(1 \times 10^{4}\right)$ were seeded in 24-well plates for cultivation. WT-circRNA_0092516 or MUTcircRNA_0092516 was constructed, and pre-NC or miR-337-3p mimic was co-transfected into 293T cells using Lipofectamine 2000 (Invitrogen). After 48 h, the cells were harvested, and then the luciferase activity was detected using the Dual-Luciferase Reporter Assay kit (Promega) according to the reagent manufacturer's instructions.

\subsection{RNA pull-down}

The primary chondrocyte lysate was incubated with biotinylated miR-337-3p at $30{ }^{\circ} \mathrm{C}$ for $30 \mathrm{~min}$. Next, the biotin-coupled RNA complex was incubated with streptavidin magnetic beads for $4 \mathrm{~h}$, and separated from the supernatant on a magnetic stand. Finally, the expression of circRNA_0092516 in the miR-337-3p pulldown complex was analyzed by qRT-PCR.

\subsection{Statistical Analysis}

SPSS 19.0 was used to analyze the statistical analysis and all data in this study were expressed as mean \pm standard deviation (SD). Differences between two groups were analyzed by Student's $t$-test, and differences among more than two groups were analyzed by one-way ANOVA. Pearson correlation coefficient analysis was used to analyze the correlation between circRNA_0092516 and miR-337-3p, circRNA_0092516, and PTEN, and the r-value was used to evaluate the correlation of two variables. A value of $P<0.05$ was considered to be statistically significant.

\section{Results}

\subsection{CircRNA_0092516 is up-regulated in tissues of osteoarthritis patients and chondrocytes stimulated by IL- $1 \beta$}


Recent studies have shown that some circRNAs are found to be dysregulated in OA and are closely related to the occurrence and development of $O A[10,21]$. Here, we detected the expression of circRNA_0092516 in OA cartilage tissues by qRT-PCR and found that circRNA_0092516 was significantly up-regulated in the OA group (Fig. 1A). Pearson correlation analysis showed that the expressions of circRNA_0092516 and miR-337-3p were negatively correlated, and the expressions of circRNA_0092516 and PTEN were positively correlated (Fig. 1B). Next, chondrocytes were isolated from normal articular cartilage tissues and then stimulated the cells with $10 \mathrm{ng} / \mathrm{mL} I \mathrm{~L}-1 \beta$ for $24 \mathrm{~h}$ to establish an in vitro OA model. As shown in Fig. 1C, circRNA_0092516 was significantly up-regulated in the IL-1 $\beta$ group. Besides, various previous studies have confirmed that matrix metallopeptidase 1 (MMP1) and matrix metallopeptidase 13 (MMP13), inflammatory factors IL-6 and TNF-a, and chondrocyte secreted Aggrecan (Aggrecan) and type II collagen (COL II) are all closely related to the occurrence and development of OA[22-24]. Western blot analysis indicated that the protein levels of OA marker molecules MMP1, MMP13, and inflammatory factors TNF- $a$, IL- 6 were markedly up-regulated in the IL-1 $\beta$ group, while the extracellular matrix metabolism-related molecules $\mathrm{COL} I I$ and Aggrecan were significantly down-regulated (Fig. 1D). Collectively, these data suggested that the abnormal expression of circRNA_0092516 might be related to the progress of $\mathrm{OA}$.

\subsection{Interference with circRNA_0092516 promotes chondrocyte proliferation and inflammatory factors release and inhibits cell apoptosis}

Next, we isolated chondrocytes from normal articular cartilage tissues and transfected sicircRNA_0092516 into chondrocytes and then stimulated the cells with $10 \mathrm{ng} / \mathrm{mL}$ IL-1 $\beta$ for $24 \mathrm{~h}$ to further explore the role of circRNA_0092516 in chondrocyte proliferation, apoptosis, and inflammatory factor release. From the results of qRT-PCR, we found that we had successfully interfered with circRNA_0092516 in chondrocytes, and IL-1 $\beta$ stimulation down-regulated the expression of miR-337-3p, while this downregulation was reversed after the interference with circRNA_0092516 (Fig. 2A). Western blot analysis indicated that the interference with circRNA_0092516 down-regulated the protein levels of MMP1, MMP13, and inflammatory factors IL- 6 and TNF-a, and up-regulated extracellular matrix metabolismrelated molecules COL II and Aggrecan (Fig. 2B). As reported, PI3K/AKT and NF-KB signaling pathways are related to $O A$, and $P T E N$ can regulate PI3K/AKT signaling pathways $[25,26]$, so we next examined the protein levels of PTEN and NF-KB p65. Also, as shown in Fig. 2C, IL-1 $\beta$ stimulation up-regulated the protein levels of PTEN and p-p65, while this up-regulation was reversed after the interference with circRNA_0092516. MTT and flow cytometry results showed that IL-1 $\beta$ stimulation inhibited the proliferation of chondrocytes (Fig. 2D) and promoted cell apoptosis (Fig. 2E), while these effects were reversed after the interference with circRNA_0092516. Besides, the analysis of Western blot revealed that IL-1 $\beta$ stimulation up-regulated the protein levels of cleaved-caspase- 3 and Bax, and down-regulated Bcl-2, while these effects were reversed after the interference with circRNA_0092516 (Fig. 2F). Overall, the above experimental results indicated that the interference with circRNA_0092516 promoted chondrocyte proliferation and inflammatory factors release and inhibited cell apoptosis. 


\section{3 circRNA_0092516 is a bait for miR-337-3p}

Next, we further explored the potential mechanism of circRNA_0092516 in OA. Bioinformatics online prediction software found that there were binding sites between circRNA_0092516 and miR-337-3p (Fig. 3A), and dual-luciferase reporter gene results indicated that miR-337-3p negatively regulated the luciferase activity of circRNA_0092516 (Fig. 3B). Besides, the results of RNA pull-down experiments revealed that circRNA_0092516 was enriched in the cell lysates pulled by miR-337-3p-Biotin (Fig. 3C), suggesting that there was an interaction between circRNA_0092516 and miR-337-3p. Next, we transfected si-circRNA_0092516 into chondrocytes and then stimulated the cells with $10 \mathrm{ng} / \mathrm{mL} \mathrm{IL}-1 \beta$ for $24 \mathrm{~h}$. As shown in Fig. 3D, the interference with circRNA_0092516 significantly up-regulated the expression of miR-337-3p. These data indicated that circRNA_0092516 could be used as a bait for miR337-3p, and circRNA_0092516 negatively regulated the expression of miR-337-3p.

\subsection{Interference with circRNA_0092516 promotes chondrocyte proliferation and inflammatory factors release and inhibits cell apoptosis through miR-337-3p}

Subsequently, we transfected si-circRNA_0092516, si-circRNA_0092516 + miR-337-3p inhibitor into chondrocytes and then stimulated the cells with $10 \mathrm{ng} / \mathrm{mL} \mathrm{IL}-1 \beta$ for $24 \mathrm{~h}$. We had previously confirmed that the interference with circRNA_0092516 down-regulated the protein levels of MMP1, MMP13, IL-6 and TNF-a, and up-regulated COL II and Aggrecan (Fig. 2B), and our further study indicated that these effects were reversed after the transfection of miR-337-3p inhibitor (Fig. 4A). Also, MTT and flow cytometry results revealed that the interference with circRNA_0092516 promoted the proliferation of chondrocytes (Fig. 2D) and repressed cell apoptosis (Fig. 2E), and our further research showed that these effects were reversed after the transfection of miR-337-3p inhibitor (Fig. 4B\& Fig. 4C). Moreover, Western blot analysis indicated that the interference with circRNA_0092516 down-regulated the protein levels of cleavedcaspase-3 and Bax, and up-regulated Bcl-2 (Fig. 2F), while these effects were reversed after the transfection of miR-337-3p inhibitor (Fig. 4D). Overall, miR-337-3p reversed the effect of the interference with circRNA_0092516 on chondrocyte proliferation, apoptosis, and inflammatory factors release.

\subsection{Interference with circRNA_0092516 affects PTEN expression and NF-KB signaling pathway through miR-337- $3 p$}

Furthermore, si-circRNA_0092516, si-circRNA_0092516 + miR-337-3p inhibitor was transfected into chondrocytes and then stimulated the cells with $10 \mathrm{ng} / \mathrm{mL}$ IL-1 $\beta$ for $24 \mathrm{~h}$. The results of qRT-PCR had indicated that the interference with circRNA_0092516 down-regulated the expression of circRNA_0092516 and up-regulated miR-337-3p (Fig. 2A), and our further research showed that these effects were reversed after the transfection of miR-337-3p inhibitor (Fig. 5A). Besides, Western blot analysis revealed that the interference with circRNA_0092516 significantly down-regulated the protein levels of PTEN and p-p65, and our further research showed that this down-regulation was reversed after the transfection of miR-337- 
3p inhibitor (Fig. 5B). The above data indicated that the interference with circRNA_0092516 regulated PTEN expression and NF-kB signaling pathway through miR-337-3p.

\section{Discussion}

At present, there is still a lack of effective strategies for the treatment of OA[27]. To explore effective treatment options, we urgently need to explore the underlying molecular mechanisms that promote the progress of OA and identify new biological targets. Here, we determined that circRNA_0092516 was a key up-regulator of circRNAs related to the progression of $O A$, and our in-depth studies found that the interference with circRNA_0092516 promoted chondrocyte proliferation and repressed cell apoptosis through miR-337-3p/PTEN axis, thereby alleviating OA.

CircRNAs are a class of endogenous non-coding RNAs that are widely distributed and characterized by tissue specificity and conservation[28]. More and more research shows that circRNAs can act as a ceRNA under different physiological and pathological conditions, and then play regulatory roles in various human diseases including $\mathrm{OA}[29,30]$. Zhang et al. found that circRNA-CDR1as acts as a sponge of microRNA-641 to regulate extracellular matrix metabolism and inflammation to promote the development of OA[31]. Also, Liu et al. found that circRNA-CER regulates MMP13 expression by competitively binding to miR-136 with MMP13, and participates in the degradation process of chondrocyte ECM in OA[32]. In this study, we found that circRNA_0092516 was markedly up-regulated in the tissues of osteoarthritis patients and chondrocytes stimulated by IL-1 $\beta$, and then we verified the interaction between circRNA_0092516 and miR-337-3p through the dual-luciferase reporter gene assay. Besides, we also found that circRNA_0092516 negatively regulated the expression of miR-337-3p. Therefore, the above data of our experiments indicated that circRNA_0092516 targeted miR-337-3p by acting as a sponge.

So far, increasing miRNAs have been found to play core functions in the pathogenesis and process of OA by interacting with the 3 'UTR of their target mRNAs [33,34]. As reported, miRNA-15a-5p regulates the development of OA by targeting PTHrP in chondrocytes[35]. Another study points out that miRNA-93 alleviates OA by targeting TLR4 to inhibit chondrocyte apoptosis and inflammation[36]. Moreover, our previous study has been found that miR-337-3p is significantly down-regulated in OA cartilage tissues[18], suggesting that miR-337-3p might be related to the progress of OA. Phosphatase and tensin homolog (PTEN) is widely regarded as a tumor suppressor and has been reported to have significantly low expression in $\mathrm{OA}[37,38]$. Importantly, our previous study also indicated that miR-337-3p is involved in the occurrence and development of OA by regulating PTEN to promote chondrocyte proliferation and inhibit apoptosis[18]. Here, our results further confirmed that the expressions of circRNA_0092516 and miR-337-3p were negatively correlated, and the expressions of circRNA_0092516 and PTEN were positively correlated, and miR-337-3p reversed the effect of the interference with circRNA_0092516 on the down-regulation of PTEN and the proliferation and apoptosis of chondrocytes and the NF-KB signaling pathway.

In summary, our research showed that circRNA_0092516 was markedly up-regulated in the tissues of osteoarthritis patients and chondrocytes stimulated by IL-1 3 , and the interference with circRNA_0092516 
promoted chondrocyte proliferation and inhibited cell apoptosis through miR-337-3p/PTEN axis, thereby improving $O A$. This might provide new strategies and directions for the treatment of $O A$, which was of great significance.

\section{Declarations}

\section{Ethics approval and consent to participate}

This study was approved by the Human Ethics Committee of the Third Affiliated Hospital of Suchow University.

\section{Consent for publication}

The study was undertaken with the patient's consent.

\section{Availability of data and materials}

Not applicable.

\section{Declarations}

\section{Ethics approval and consent to participate}

This study was approved by the Human Ethics Committee of the Third Affiliated Hospital of Suchow University.

\section{Consent for publication}

The study was undertaken with the patient's consent.

\section{Availability of data and materials}

Not applicable.

\section{Conflict of Interest}

The authors declare that they have no conflict of interest.

\section{Competing Interests}

The authors declare that they have no competing interests.

\section{Funding}

This work was supported by the Major Science and Technology Project of Changzhou Health Commission (ZD201908). 


\section{Authors' contributions}

ZH conceived and designed the study and drafted the manuscript. WM collected the data and JX contributed to the statistical analysis. XD interpreted the data. WL put forward the concept of the study and reviewed the manuscript. All authors read and approved the final manuscript.Acknowledgements

We would like to express our gratitude to all those who financed the subject.

\section{References}

1. Cross $M$, et al. The global burden of hip and knee osteoarthritis: estimates from the global burden of disease 2010 study. Ann Rheum Dis. 2014;73(7):1323-30.

2. Gregori D, et al. Association of Pharmacological Treatments With Long-term Pain Control in Patients With Knee Osteoarthritis: A Systematic Review and Meta-analysis. Jama. 2018;320(24):2564-79.

3. Bijlsma JW, Berenbaum F, Lafeber FP. Osteoarthritis: an update with relevance for clinical practice. Lancet. 2011;377(9783):2115-26.

4. Hwang HS, Kim HA. Chondrocyte Apoptosis in the Pathogenesis of Osteoarthritis. Int J Mol Sci. 2015;16(11):26035-54.

5. Huang $Y$, et al. TAR DNA-binding protein 43 inhibits inflammatory response and protects chondrocyte function by modulating RACK1 expression in osteoarthritis. Biomed Pharmacother. 2017;85:362-71.

6. Szabo L, Salzman J. Detecting circular RNAs: bioinformatic and experimental challenges. Nat Rev Genet. 2016;17(11):679-92.

7. Memczak S, et al. Circular RNAs are a large class of animal RNAs with regulatory potency. Nature. 2013;495(7441):333-8.

8. Li Z, et al., Circ_0136474 and MMP-13 suppressed cell proliferation by competitive binding to miR127-5p in osteoarthritis. 2019. 23(10): p. 6554-6564.

9. Shen S, et al., CircSERPINE2 protects against osteoarthritis by targeting miR-1271 and ETS-related gene. 2019. 78(6): p. 826-836.

10. Li HZ, et al., The potential roles of circRNAs in osteoarthritis: a coming journey to find a treasure. Biosci Rep, 2018. 38(5).

11. Hansen TB, et al. Natural RNA circles function as efficient microRNA sponges. Nature. 2013;495(7441):384-8.

12. Zheng Q, et al. Circular RNA profiling reveals an abundant circHIPK3 that regulates cell growth by sponging multiple miRNAs. Nat Commun. 2016;7:11215.

13. Bartel DP. MicroRNAs: target recognition and regulatory functions. Cell. 2009;136(2):215-33.

14. Lu J, et al. MicroRNA-218-5p as a Potential Target for the Treatment of Human Osteoarthritis. Mol Ther. 2017;25(12):2676-88. 
15. Zhang $\mathrm{X}$, et al., miR-146a facilitates osteoarthritis by regulating cartilage homeostasis via targeting Camk2d and Ppp3r2. 2017. 8(4): p. e2734.

16. Xue J, et al. The hsa-miR-181a-5p reduces oxidation resistance by controlling SECISBP2 in osteoarthritis. BMC Musculoskelet Disord. 2018;19(1):355.

17. Chen L, Li Q, Wang J, MiR-29b-3p promotes chondrocyte apoptosis and facilitates the occurrence and development of osteoarthritis by targeting PGRN. 2017. 21(12): p. 3347-3359.

18. Huang Z, et al. MiR-337-3p promotes chondrocytes proliferation and inhibits apoptosis by regulating PTEN/AKT axis in osteoarthritis. Biomed Pharmacother. 2017;95:1194-200.

19. Hu C, Sun Y, Yang X. Pioglitazone up-regulates MALAT1 and promotes the proliferation of endothelial progenitor cells through increasing c-Myc expression in type 2 diabetes mellitus. Aging Pathobiology Therapeutics. 2020;2(1):38-44.

20. Increased LncRNA. PVT-1 is associated with tumor proliferation and predicts poor prognosis in cervical cancer. Clinical surgery research communications. 2017;1(1):10-7.

21. Zhou ZB, et al., circRNA.33186 Contributes to the Pathogenesis of Osteoarthritis by Sponging miR127-5p. Mol Ther, 2019. 27(3): p. 531-541.

22. Goldring MB, Goldring SR. Osteoarthritis J Cell Physiol. 2007;213(3):626-34.

23. Bhosale AM, Richardson JB. Articular cartilage: structure, injuries and review of management. Br Med Bull. 2008;87:77-95.

24. Vincenti MP, Brinckerhoff CE. Transcriptional regulation of collagenase (MMP-1, MMP-13) genes in arthritis: integration of complex signaling pathways for the recruitment of gene-specific transcription factors. Arthritis Res. 2002;4(3):157-64.

25. Xie Y, et al. Power of PTEN/AKT: Molecular switch between tumor suppressors and oncogenes. Oncol Lett. 2016;12(1):375-8.

26. Kim JH, et al. The non-provitamin A carotenoid, lutein, inhibits NF-kappaB-dependent gene expression through redox-based regulation of the phosphatidylinositol 3-kinase/PTEN/Akt and NFkappaB-inducing kinase pathways: role of $\mathrm{H}(2) \mathrm{O}(2)$ in NF-kappaB activation. Free Radic Biol Med. 2008;45(6):885-96.

27. Berenbaum F. Osteoarthritis year 2010 in review: pharmacological therapies. Osteoarthritis Cartilage. 2011;19(4):361-5.

28. Patop IL, Wüst S, Kadener S. Past, present, and future of circRNAs. 2019. 38(16): p. e100836.

29. Xiao K, et al. Identification of differentially expressed long noncoding RNAs in human knee osteoarthritis. J Cell Biochem. 2019;120(3):4620-33.

30. He J, et al. Circular RNAs and cancer. Cancer Lett. 2017;396:138-44.

31. Zhang W, et al. Circular RNA-CDR1as acts as the sponge of microRNA-641 to promote osteoarthritis progression. J Inflamm (Lond). 2020;17:8.

32. Liu Q, et al. Circular RNA Related to the Chondrocyte ECM Regulates MMP13 Expression by Functioning as a MiR-136 'Sponge' in Human Cartilage Degradation. Sci Rep. 2016;6:22572. 
33. Kopańska M, et al. MiRNA expression in the cartilage of patients with osteoarthritis. J Orthop Surg Res. 2017;12(1):51.

34. Swingler TE, et al. The function of microRNAs in cartilage and osteoarthritis. Clin Exp Rheumatol. 2019;37(5):40-7., , Suppl 120.

35. Duan ZX, Huang P. MicroRNA-15a-5p Regulates the Development of Osteoarthritis by Targeting PTHrP in Chondrocytes. 2019. 2019: p. 3904923.

36. Ding $Y$, et al. MicroRNA-93 inhibits chondrocyte apoptosis and inflammation in osteoarthritis by targeting the TLR4/NF-KB signaling pathway. Int J Mol Med. 2019;43(2):779-90.

37. Chen L, Guo D. The functions of tumor suppressor PTEN in innate and adaptive immunity. Cell Mol Immunol. 2017;14(7):581-9.

38. Iwasa $\mathrm{K}$, et al. PTEN regulates matrix synthesis in adult human chondrocytes under oxidative stress. J Orthop Res. 2014;32(2):231-7.

\section{Figures}

A

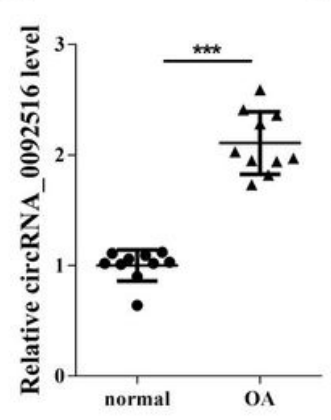

B

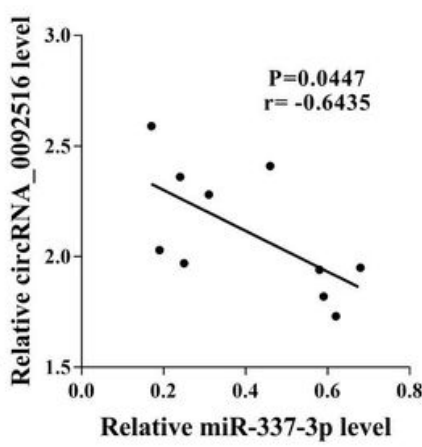

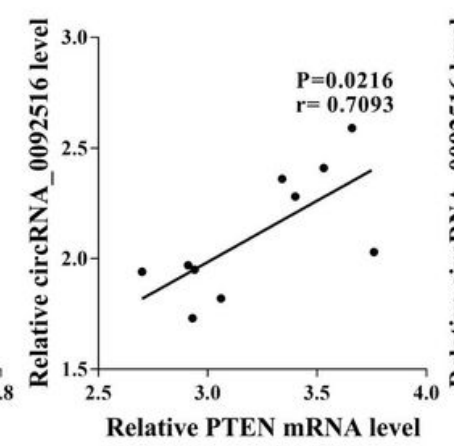

C

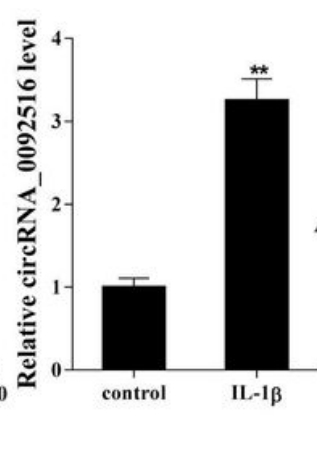

D

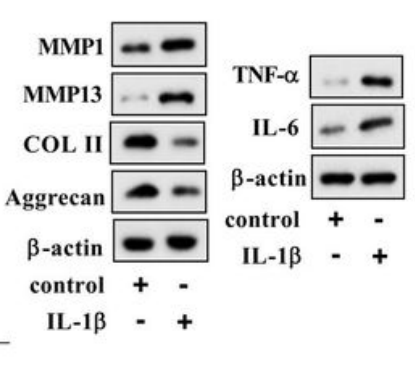

\section{Figure 1}

Expression of circRNA_0092516 in the tissues of osteoarthritis patients and chondrocytes stimulated by IL-13. (A) qRT-PCR was used to detect the expression of circRNA_0092516 in OA cartilage tissues. (B) Pearson correlation analysis was used to analyze the correlation between the expressions of circRNA_0092516 and miR-337-3p, and the expressions of circRNA_0092516 and PTEN. Next, chondrocytes were isolated from normal articular cartilage tissues and then stimulated the cells with 10 $\mathrm{ng} / \mathrm{mL}$ IL-1 $\beta$ for $24 \mathrm{~h}$ to establish an in vitro OA model. (C) Detection of circRNA_0092516 expression. (D) Western blot was used to detect the protein levels of OA marker molecules matrix metallopeptidase 1 (MMP1) and matrix metallopeptidase 13 (MMP13), extracellular matrix metabolism-related molecules COL II and Aggrecan, and inflammatory factors TNF-a, IL-6. ${ }^{* \star *} \mathrm{P}<0.001 \mathrm{vs}$. normal group. ${ }^{* *} \mathrm{P}<0.01 \mathrm{vs}$. control group. 
A

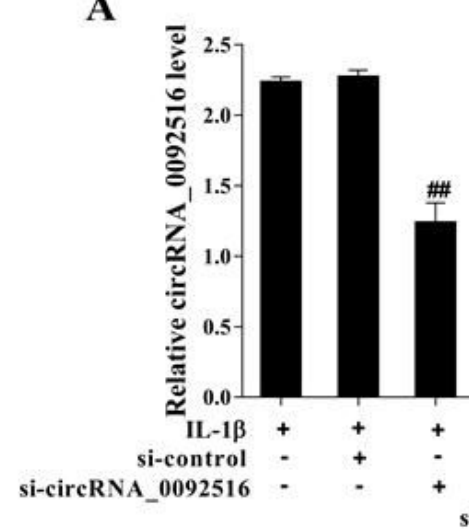

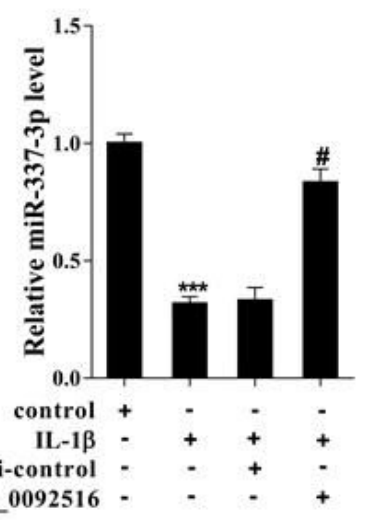
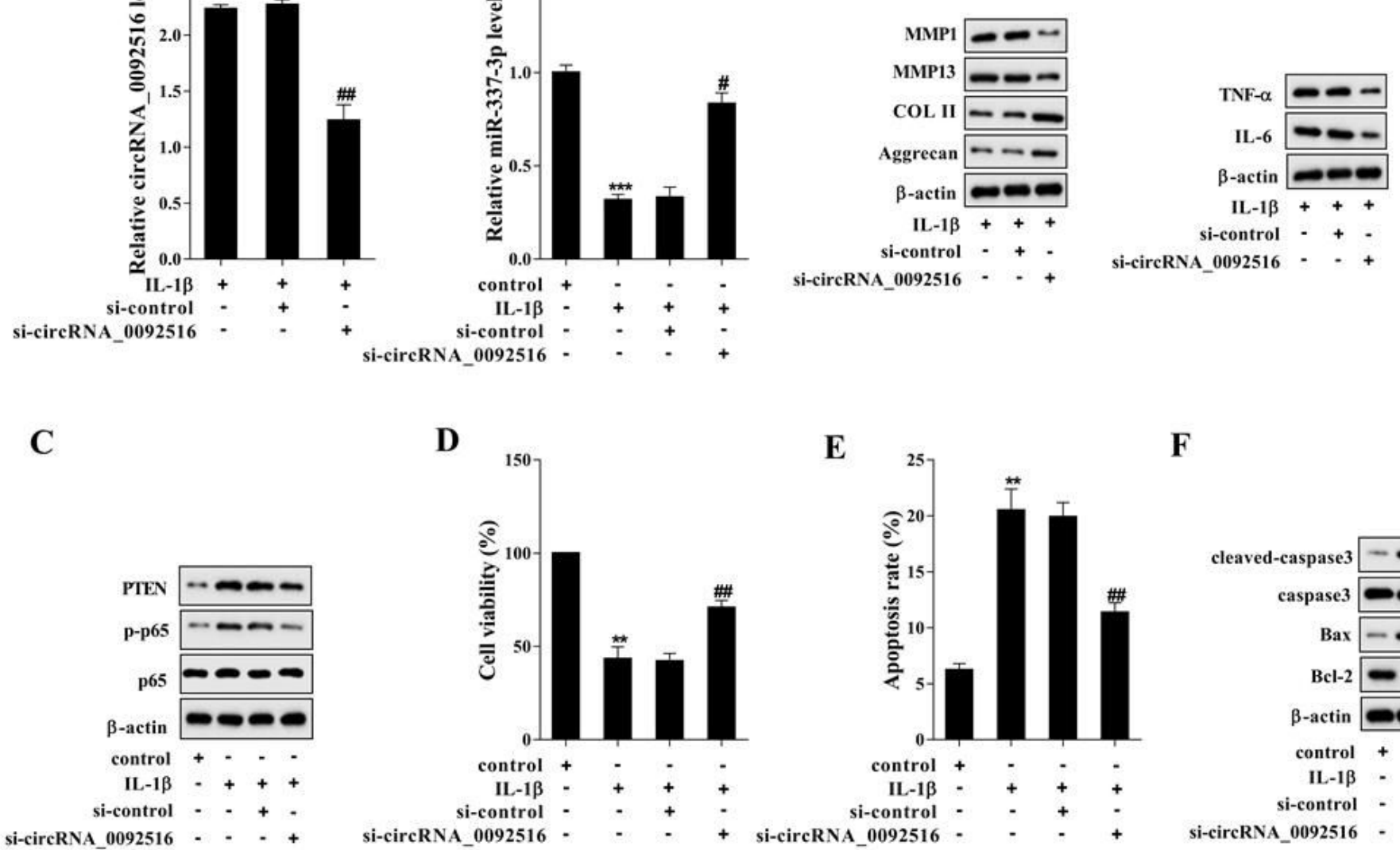

F

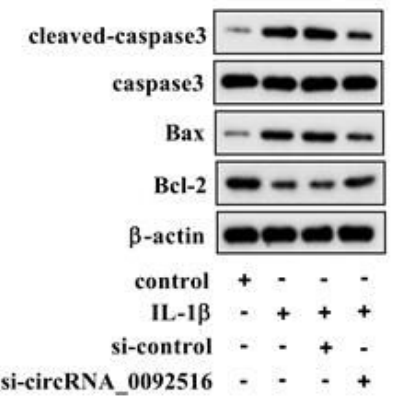

\section{Figure 2}

Effect of circRNA_0092516 on chondrocyte proliferation, apoptosis, and inflammatory factors release. Chondrocytes were isolated from normal articular cartilage tissues and transfected si-circRNA_0092516 into chondrocytes and then stimulated the cells with $10 \mathrm{ng} / \mathrm{mL} \mathrm{IL}-1 \beta$ for $24 \mathrm{~h}$. (A) Detection of circRNA_0092516 and miR-337-3p expressions. (B) Detection of the protein levels of MMP1, MMP13, and inflammatory factors IL-6 and TNF-a, and extracellular matrix metabolism-related molecules COL II and Aggrecan. (C) Detection of the protein levels of PTEN, p-p65, and p65. (D) MTT was used to detect the proliferation of chondrocytes. (E) Flow cytometry was used to detect the apoptosis of chondrocytes. (F) Detection of the protein levels of cleaved-caspase-3, caspase3, Bax, and Bcl-2. \#P<0.05, \#\#P<0.01 vs. IL$1 \beta+$ si-control group. ${ }^{*} \mathrm{P}<0.01,{ }^{*} * \mathrm{P}<0.001$ vs. control group. 

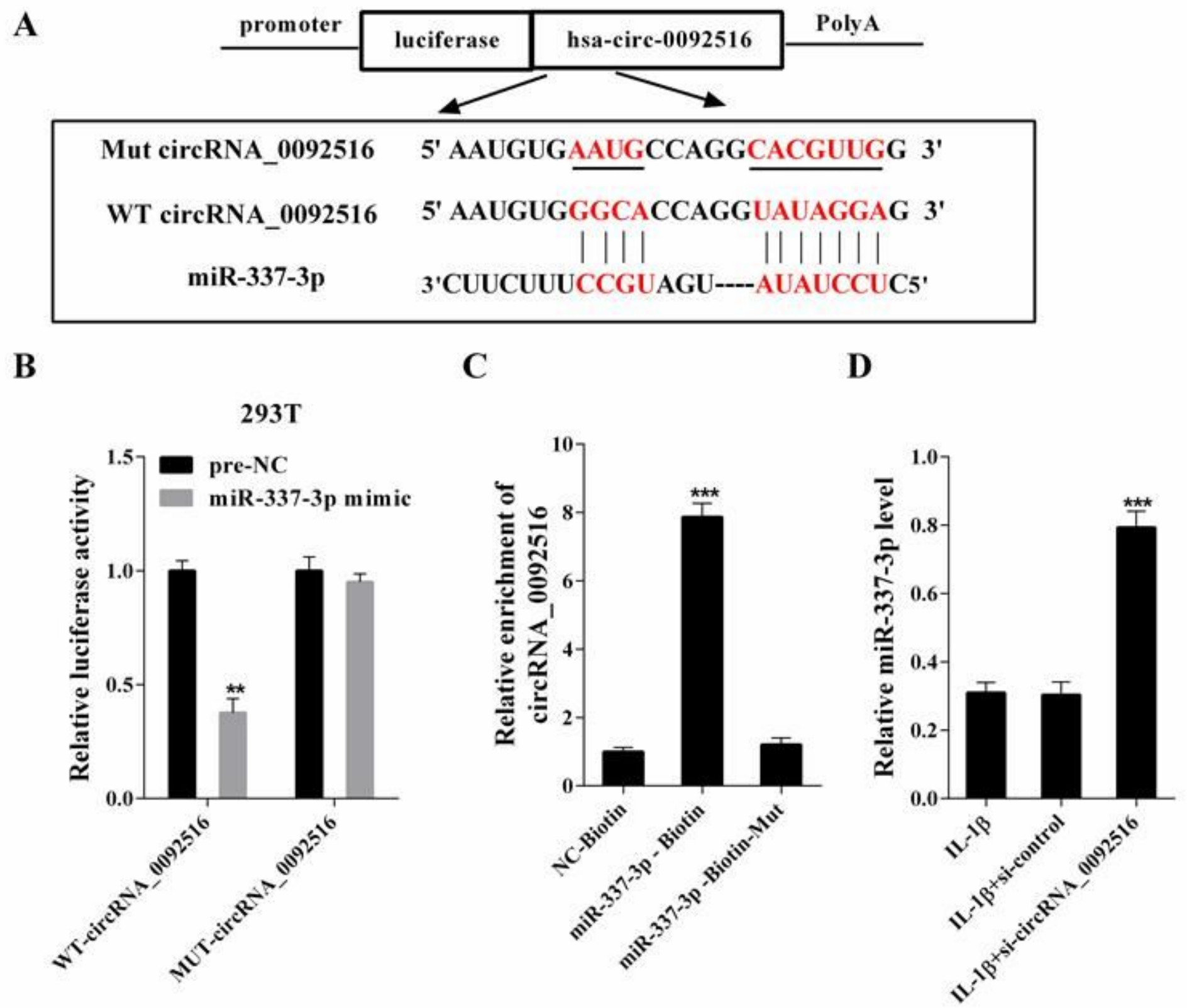

Figure 3

Interaction between circRNA_0092516 and miR-337-3p. (A) Bioinformatics online prediction software was used to analyze the binding sites between circRNA_0092516 and miR-337-3p. (B) Dual-luciferase reporter gene assay was used to detect the effect of miR-337-3p on the luciferase activity of circRNA_0092516.

(C) RNA pull-down experiment was used to analyze the interaction between circRNA_0092516 and miR337-3p. Next, si-circRNA_0092516 was transfected into chondrocytes and then stimulated the cells with $10 \mathrm{ng} / \mathrm{mL} \mathrm{IL}-1 \beta$ for $24 \mathrm{~h}$. (D) Detection of miR-337-3p expression. ${ }^{*} \mathrm{P}<0.01$ vs. pre-NC group. ${ }^{\star * *} \mathrm{P}<0.001$ vs. NC-Biotin or IL-1 $\beta+$ si-control group. NC: negative control. 


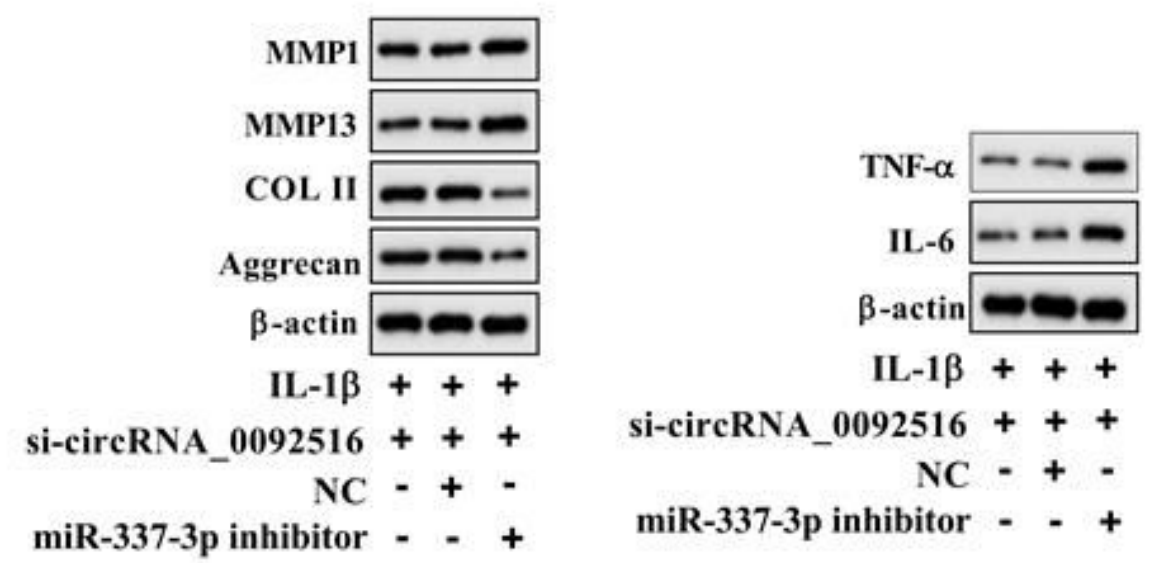

B

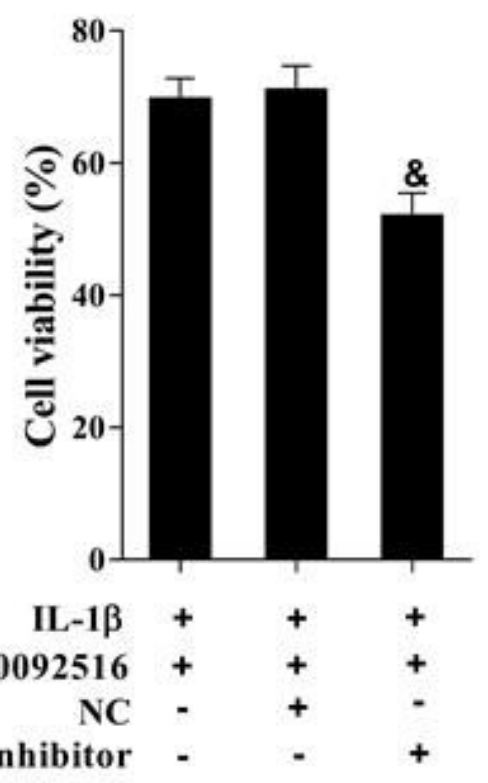

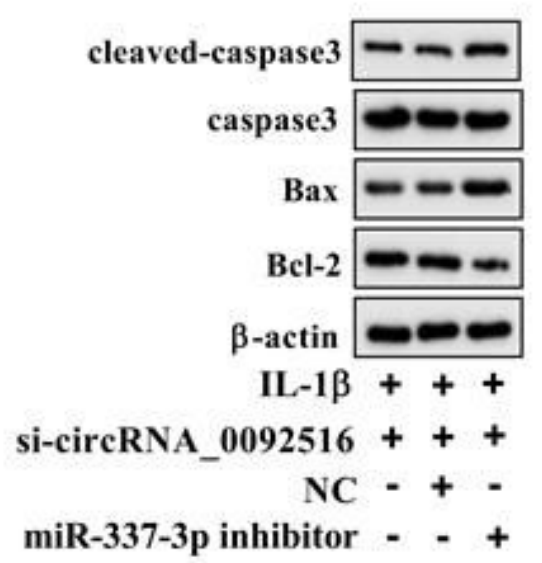

C

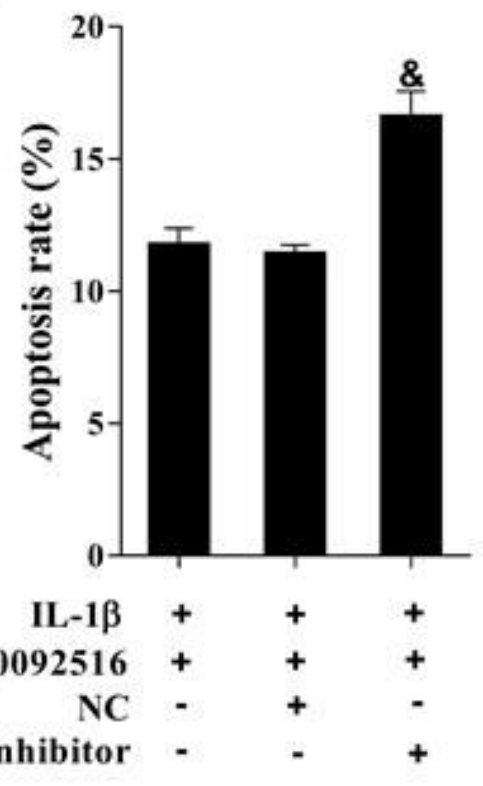

Figure 4

circRNA_0092516 affects chondrocyte proliferation, apoptosis and inflammatory factors release through miR-337-3p. si-circRNA_0092516, si-circRNA_0092516 + miR-337-3p inhibitor was transfected into chondrocytes and then stimulated the cells with $10 \mathrm{ng} / \mathrm{mL} \mathrm{IL}-1 \beta$ for $24 \mathrm{~h}$. (A) Detection of the protein levels of MMP1, MMP13, IL-6, TNF- $a$, COL II, and Aggrecan. (B) Detection of the proliferation of chondrocytes. (C) Detection of the apoptosis of chondrocytes. (D) Detection of the protein levels of cleaved-caspase-3, caspase3, Bax, and Bcl-2. \&P<0.05 vs. IL-1 $\beta$ + si-circRNA_0092516 + NC group. NC: negative control. 
A

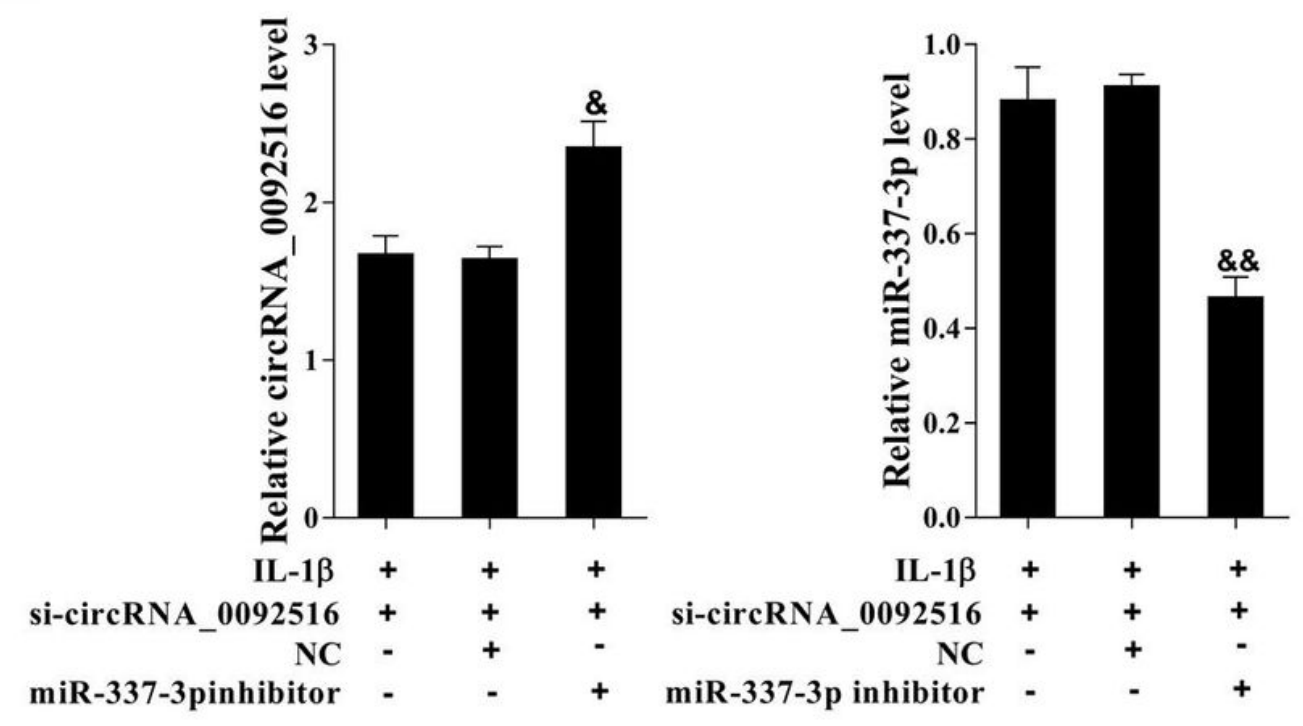

B

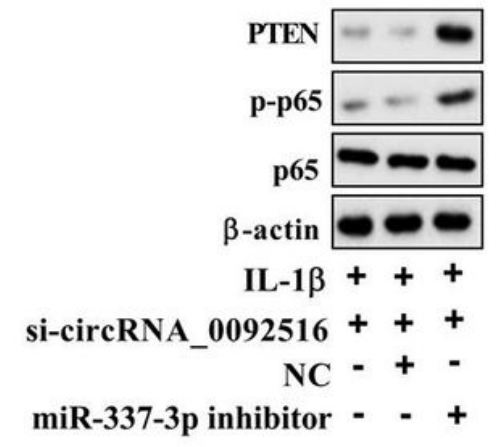

Figure 5

Effect of circRNA_0092516 on PTEN expression and NF-KB signaling pathway through miR-337-3p. sicircRNA_0092516, si-circRNA_0092516 + miR-337-3p inhibitor was transfected into chondrocytes and then stimulated the cells with $10 \mathrm{ng} / \mathrm{mL}$ IL-1 $\beta$ for $24 \mathrm{~h}$. (A) Detection of circRNA_0092516 and miR-337$3 p$ expressions. (B) Detection of the protein levels of PTEN, p-p65 and p65. \&P<0.05, \&\&P<0.01 vs. IL-1 $\beta+$ si-circRNA_0092516 + NC group. NC: negative control. 